\title{
A double-blind randomised controlled trial of gloved versus ungloved merocel middle meatal spacers for endoscopic sinus surgery*
}

\author{
Elaheh Akbari', Carl M. Philpott' ${ }^{1}$ Avi J. Ostry², Allan Clark³, Amin R. Javer \\ St. Paul's Sinus Centre, St. Paul's Hospital, Vancouver, British Columbia, Canada \\ Department of Pathology, St. Paul's Hospital, Vancouver, British Columbia, Canada \\ School of Medicine, Health Policy and Practice, University of East Anglia, Norwich, United Kingdom
}

Rhinology 50: 306-310, 2012

DOI: 10.4193/Rhino11.215

*Received for publication:

September 16,2010

Accepted: February 20, 2012

\section{Summary}

Background: Middle meatal spacers are commonly used following endoscopic sinus surgery to prevent post-operative bleeding and lateralization of the middle turbinates. The effects of nasal packing on post-operative sinonasal mucosal healing remain unknown in humans.

Objective: This study aims to compare the histopathalogical effects of Merocel and Merocel covered with a finger glove on mucosal healing, and patients' discomfort immediately post-operatively after endoscopic sinus surgery and at removal of the nasal packing.

Methods: Thirty-seven patients with chronic rhinosinusitis undergoing bilateral endoscopic sinus surgery were enrolled in a prospective study. Patients were randomized and blinded to receive Merocel middle meatal spacer (MMMS) in one nostril and finger glove Merocel middle meatal spacer (FGMMS) in the contra lateral side. Patients were seen on post-operative day 6 , and completed a visual analogue score reporting the post-operative discomfort from nasal packing on each side. Following the removal of nasal packing, patients indicated which side caused more discomfort on removal. Biopsies were taken from the middle turbinates and sent to a blinded pathologist who scored the level of mucosal inflammation from 0 - 4.

Results: There was no statistically significant difference between MMMS and FGMMS in regards to their effect on sinonasal mucosal inflammation and discomfort post-operatively. A statistically significant difference was noted with respect to discomfort at removal with the uncovered Merocel more likely to cause discomfort when compared to the Merocel covered in a glove finger.

Conclusion: MMMS and FGMMS are equivalent in the amount of sinonasal mucosal inflammation and discomfort post endoscopic sinus surgery. However, the main advantage of the FGMMS was a significant reduction in pain on removal when compared with the MMMS.

Key words: middle meatal spacer, nasal packing, endoscopic sinus surgery, randomized trial

\section{Introduction}

Functional endoscopic sinus surgery (FESS) has become an effective management for patients with recurrent acute rhinosinusitis (RARS) and chronic rhinosinusitis (CRS) refractory to medical therapy. The goal of surgery is to re-establish ventilation and drainage via the natural ostia, causing minimal damage to the sinonasal mucosa.
Middle meatal packing is commonly used post-operatively following FESS to control bleeding, prevent lateralization of the middle turbinate and synechiae formation or restenosis ${ }^{(1)}$. Various types of middle meatal spacers (MMS) including removable nasal packing and absorbable packs have been developed ${ }^{(2-5)}$. Although many of these materials have proven to be effective hemostats, data are conflicting regarding their 
effect on mucosal healing, and there is a paucity of prospective randomized trials in humans assessing the effects of MMS on mucosal healing. Thus, practices regarding the use MMS after endoscopic sinus surgery vary widely among surgeons. A recent study by Titiz et al., ${ }^{(6)}$ showed that there was a greater degree of damage to the lamina propria of the septal mucosa of rabbits when Merocel was directly applied to the nasal mucosa of rabbits as compared to Merocel in a glove finger. We sought to replicate this study in human subjects.

The objective of this study was 1) to compare the histopathological effects of Merocel MMS (MMMS) and Merocel covered with a finger glove (FGMMS) on mucosal healing, and 2) to determine the patients' discomfort post-operatively after FESS and at removal of the MMS.

\section{Materials and methods}

The approval of Clinical Research Ethics Board of University of British Columbia was obtained for the study. All study subjects provided informed consents.

\section{Surgery}

Thirty-seven consecutive patients undergoing bilateral computer assisted endoscopic sinus surgery (BiCASS) for chronic rhinosinusitis were enrolled in a prospective study at the St. Paul's Sinus Centre in Canada, Vancouver, British Columbia. Patient inclusion criteria included all patients undergoing bilateral endoscopic sinus surgery in whom spacers would be placed lateral to the middle turbinates as a matter of operative routine. Exclusion criteria included patients under the age of 19, patients unable to understand English, unilateral sinus surgery or surgery for neoplastic disease, known bleeding disorders and any cases where spacers are not used.

All patients started a course of Co-amoxiclav $(875 \mathrm{mg}$ PO bd for two weeks) and Prednisolone (20 mg PO od for one week, and $10 \mathrm{mg}$ PO od for one week) starting one week prior to their operation and continued for one week post-operatively. Patients were randomized using a closed envelope system to receive the MMMS (a nonabsorbable foam polymer of hydroxylated polyvinyl acetal) in one middle meatus (right or left), and the FGMMS in the contra-lateral middle meatus intra-operatively. At the end of surgery, an envelope was opened and the appropriate spacers were then placed lateral to the middle turbinates according to the randomization. Patients were under a general anesthetic when the MMSs were placed and were therefore blinded to the type of packing on each side. A digital photograph of the spacer in place was taken for each patient once the MMSs were in place (Figure 1).

\section{Follow-up}

All patients were seen in follow up on post-operative day 6. Patients were asked to make a mark on a visual analogue scale $(0-10 \mathrm{~cm})$ to demonstrate the amount of discomfort they were experiencing from each side of the nose as a result of the MMS being present during the past 6 days. The MMSs were then removed and the patients were asked which MMS caused more discomfort upon removal. At the same visit, a small mucosal biopsy was taken from the lateral surface of each middle turbinates as confirmed by a demarcation on an endoscopic photograph taken during the procedure.

\section{Histology}

Tissue specimens were fixed in buffered $10 \%$ formaldehyde solution at room temperature. Biopsy specimens were then processed routinely for light microscopy. Three $\mu \mathrm{m}$ sections were cut from the paraffin blocks and were stained with hematoxylin and eosin. Prepared slides were sent to a pathologist (AJO) who was blinded to the randomization of the MMSs for histopathological examination.

The pathologist devised a 0 - 4 scoring system to objectively grade the level of mucosal inflammation (Figure 2). The scores were given as follows: 0 ) normal tissue, 1 ) sub-mucosal inflammation with no inflammatory infiltrate into the epithelium, 2) inflammatory infiltrate with neutrophils into the epithelium, with no evidence of necrosis, 3 ) inflammatory infiltrate with neutrophils into the epithelium, with evidence of focal necrosis, and 4) inflammatory infiltrate with neutrophils into the epithelium, with evidence of extensive necrosis.

\section{Statistical analysis}

Assuming a $20 \%$ difference between the two sides, a minimum of 31 patients needed to be recruited to have a power of $80 \%$ using McNemar's test. Statistical analyses of continuous outcome measures were compared between MMMS, and FGMMS by carrying out a paired t-test, or a non-parametric equivalent if the assumptions of the paired t-test were not met.

\section{Results}

A total of 37 volunteer patients were recruited and randomized with a mean age of 49.6 years (range 21 - 76 years). Twenty of the patients were males (54\%) and seventeen of the patients were females (46\%). The MMMS was randomized to the left nostril of 22 patients (59\%) and FGMMS in the right nostril and the other 15 patients vice-versa. Four patients were excluded from the study: one patient withdrew her consent for biopsy, one patient's MMMS fell out on post-operative day 2 , and two patients had insufficient mucosal biopsy.

The mean pre-operative Lund-MacKay CT score [0 - 12] was 7.42 for the MMMS side, and 7.67 for the FGMMS side. No statistically significant difference was found between the MMMS and the FGMMS sides in the Lund-Mackay CT score, with a mean difference of -0.25 (95\% Cl: $-0.67,0.17, \mathrm{p}=0.24)$. 

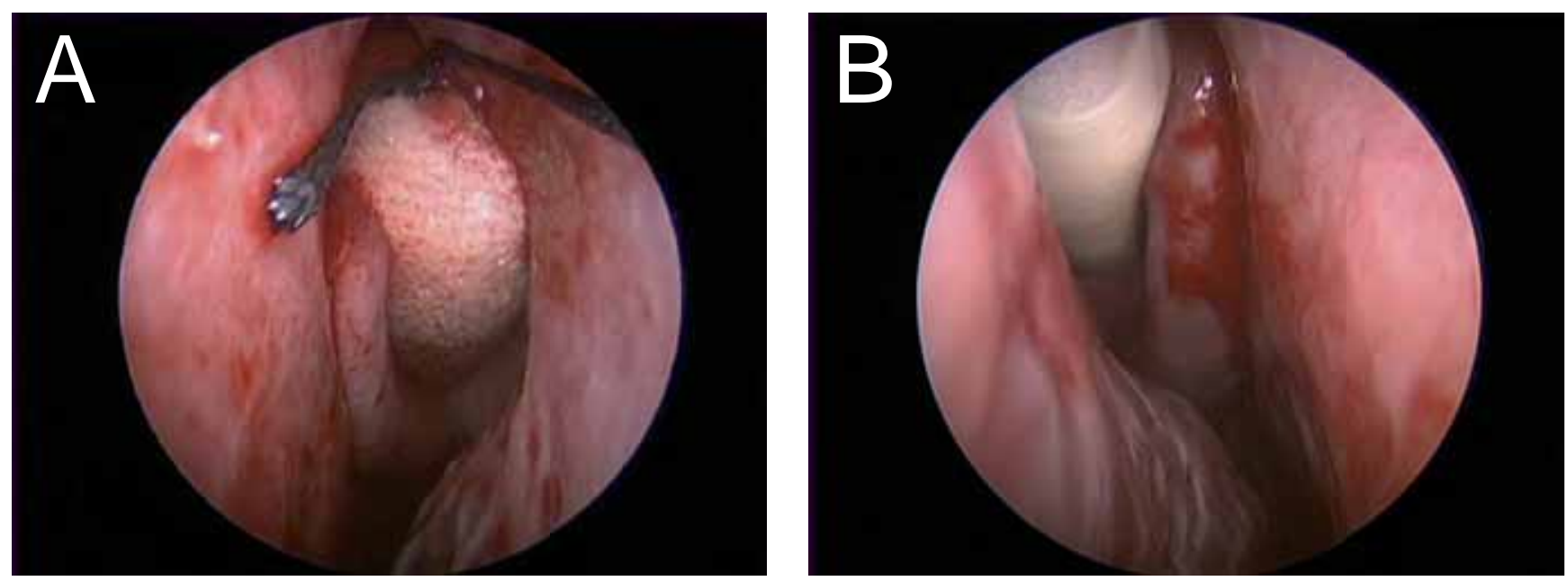

Figure 1. Intraoperative endoscopic confirmation of middle meatal spacer placement.

(A) Merocel middle meatal spacer in situ. (B) Finger glove middle meatal spacer in situ.

There was no statistically significant difference in the histopathological scores [0 - 4] between the MMMS (mean score of 2.70) and the FGMMS sides (mean score of 2.70) with a mean difference of 0 (95\% $\mathrm{Cl}:-1,0, \mathrm{p}=0.23)$.

The Spearman's correlation between the histopathological and Lund-Mackay scores for the MMMS and the FGMMS sides were $0.36(p=0.052)$ and $0.49(p=0.49)$, respectively.

The patients' mean visual analogue scores [0 - 10] for the degree of discomfort that resulted from the MMMS, and the FGMMS while in situ were 2.08 and 2.00 , respectively. There was no statistically significance in the mean difference of 0.08 (95\% Cl: - 0.89, 1.06, $\mathrm{p}=0.86$ ).

However, a statistically significant difference was found with regards to MMMS being more likely to cause more discomfort at removal when compared to the FGMMS. In total, 93\% of patients ( $95 \% \mathrm{Cl}: 76 \%, 99 \%, \mathrm{p}<0.00001)$ reported more discomfort upon removal of the MMMS.

\section{Discussion}

Mucosal preservation during FESS and good post-operative care are described as important factors in the prevention of post-operative synechiae formation and optimization of mucosal healing ${ }^{(7-9)}$. The use of MMS post-FESS is a common practice amongst sinus surgeons to primarily, prevent middle turbinate lateralization and prevent synechiae formation, but also to provide homeostasis and prevent blood or mucus accumulation in the ethmoid cavity, and theoretically this should reduce the risk of re-stenosis ${ }^{(10,11)}$. Potential complications of MMS include pack dislodgement and aspiration, obstructive sleep apnea secondary to nasal obstruction, myospherulosis, toxic shock syndrome, and foreign body granuloma. The two major disadvantages to MMS are potential mucosal injury and patient discomfort associated with the packing and its removal ${ }^{(12-14)}$.

The effect of absorbable and non-absorbable nasal packing on hemostasis, prevention of adhesions, synechia formation, and discomfort associated with packing removal has been studied ${ }^{(15)}$. Baumann and Caversaccio conducted a nonrandomized cohort study with FloSeal (a gel with collagen derived particles and topical thrombin) and Merocel MMSs and found rapid and equal intraoperative hemostasis in both groups ${ }^{(16)}$. Shoman et al., compared NasoPore (an absorbable polyurethane foam) with FGMMS in a prospective, double-blind, randomized trial of thirty patients and found no statistically significant difference between both groups with regard to patients' pain, bleeding, or discomfort on packing removal. However, they showed a statistically significant better endoscopically assessed mucosal healing associated with FGMMS ( $p=0.03$ ) four weeks post-operatively, an effect that disappeared tweleve weeks post-operatively ${ }^{(17)}$.

A previous double-blind randomized controled trial of twenty consecutive patients by Chandra et al., in 2003 showed increased adhesions $(p=0.007)$ and granulation tissue $(p=0.006)$ in ethmoid cavities packed with FloSeal compared with the cavities packed with thrombin-soaked Gelofoam at the 6-8 weeks post FESS follow-up ${ }^{(18)}$. Chandra et al., published a retrospective study of the same patient cohort in 2005 determining the long-term effects of FloSeal packing post FESS. They found a statistically significant higher incidence of adhesions ( $p=0.013$ ) and higher number of patients requiring lysis of adhesions $(p=0.046)$ in the FloSeal group during 

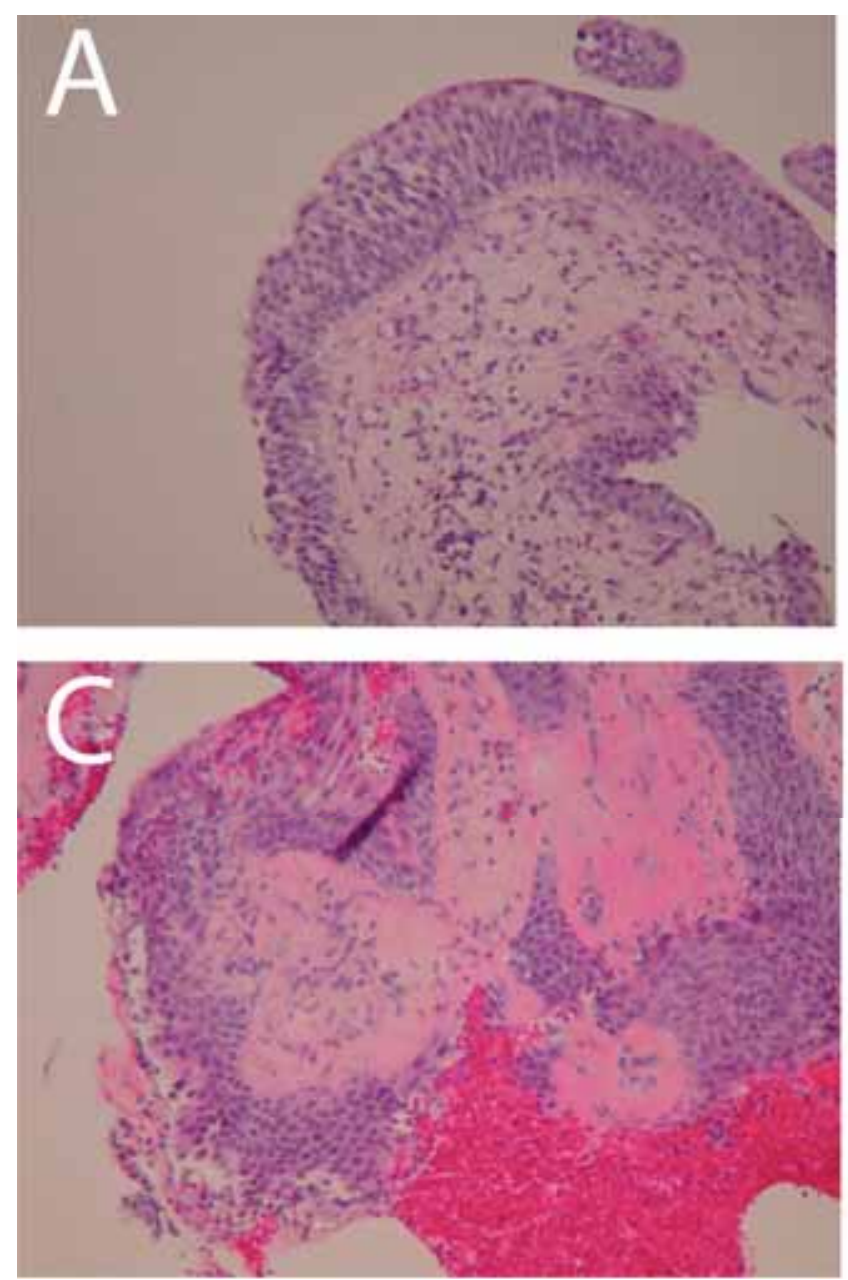
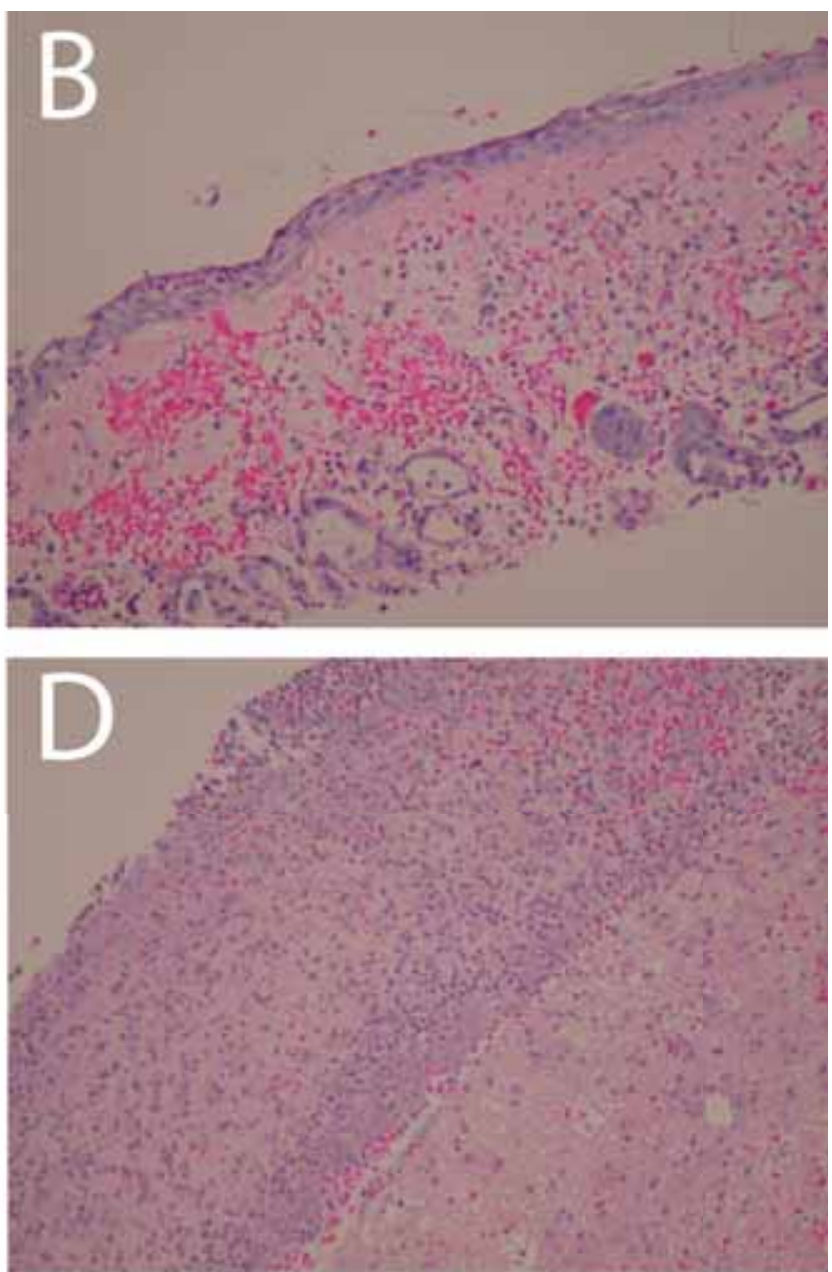

Figure 2. Histopathological scoring of sinonasal inflammation. Hematoxylin-eosin stain, 10x10 magnification. (A) Sub-mucosal inflammation with no inflammatory infiltrate into the epithelium. (B) Inflammatory infiltrate with neutrophils into the epithelium with no evidence of necrosis. (C) Inflammatory infiltrate with neutrophils into the epithelium with evidence of focal necrosis. (D) Inflammatory infiltrate with neutrophils into the epithelium with evidence of extensive necrosis.

the mean follow-up period of 21.4 months ${ }^{(19)}$. In a blinded, randomized controlled tiral of 37 patients Miller et al., did not find any statistically significant difference between MeroGel (woven hyaluronic acid) and Merocel MMSs in synechia formation after FESS ${ }^{(20)}$.

The histopathological effect of MMSs has been previously studied in animals. Robinson et al., studied the effect of topical prednisolone on sinus mucosal healing in a sheep animal model (21). They concluded that hyaluronic acid nasal packs soaked in prednisolone did not improve the speed of mucosal healing or re-ciliation. In 2005, McIntosh et al., studied the effect of Merocel packing on the healing of the nasal mucosa of sheep. They found no significant differnece between the packed and control sides in the rate of re-epithelialization, the total amount of surface cilia coverage, and the maturity of the cilia ${ }^{(22)}$. In a recent study, Titiz et al., evaluated the histopathological effects of Merocel and glove finger tampons on the nasal mucosa of rabbits ${ }^{(6)}$. They found the two types of tampons resulted in similar degrees of cilia loss in the mucosa; however, when Merocel was applied directly, it inflicted more damage on the lamina propria than glove finger tampons.

There is a lack of literature on histopathological effects of MMSs on mucosal healing in humans post FESS. To our knowledge, we conducted the first prospective, double-blind, randomized controlled trial to study the primary outcome of histopathological changes related to the use of MMMS and FGMMS on patients' sinonasal mucosa post FESS. In addition, the patients' degree of discomfort associated post-operatively from the nasal packs, and during their removal was compared between the two MMSs.

In this study, there was no statistically significant correlation between the two groups in histopathological scores and 
pre-operative Lund-Mackay CT scores. Of note, Spearman's correlation between the histopathological scores and the Lund-Mackay CT scores of both MMS types was not found to be statistically significant. This indicates that the histopathological scores reflected post-operative mucosal healing as opposed to the level of pre-operative inflammation. One of the strength of this study as compared to the previous studies evaluating the histopathological effects of MMSs on sinonasal mucosa was the scoring system used. It standardized the pathological report, and provided the pathologist with an objective way of evaluating the degree of inflammation caused by each MMS. The histopathology specimens were somewhat dependent on the operator's choice of biopsy site as histology would vary from site to site, however the authors ARJ and CMP were careful in selecting a site anteriorly on the lateral surface that had been in contact with the MMS. In addition, a portion of the surface epithelium was metaplastic squamous as opposed to native respiratory secondary to the reparative process.
The patients' discomfort associated with the MMMS and the FGMMS was found to be similar. Our study underlines work by Rudy et al., ${ }^{(23)}$ who similarly did not find a statistically significant difference in nasal obstruction, patient comfort, post-operative bleeding, nasal crusting, and post-operative adhesions when they compared the MMMS and the FGMMS. The fact that patients found the FGMMS more comfortable to have removed is important given that previous studies have demonstrated patients undergoing FESS often consider packing removal to be the most uncomfortable part of their peri-operative experience ${ }^{(3,24)}$.

\section{Conclusion}

The use of FGMMS does not convey any advantage over MMMS in terms of post-operative healing, but for the fact that it aids patient comfort on removal, the FGMMS should be the spacer of choice.

\section{Disclaimer}

No conflict of interest.

\section{REFERENCES}

1. Weber R, Keerl R, Hochapfel F, et al. Packing in endonasal surgery. Am J Otolaryngol. 2001; 22: 306-320.

2. Garth RJN, Brightwell AP. A comparison of packing materials used in nasal surgery. J Laryngol Otol. 1994; 108: 564-566.

3. Shoenberg MV, Robinson P, Ryan R. Nasal packing after routine nasal surgery-is it justified? J Laryngol Otol. 1993; 107: 902-905.

4. Genc E, Ergin NT, Bilezikci B. Comparison of suture and nasal packing in rabbit noses. Laryngoscope. 2004; 114: 639-645.

5. Chevillard C, Rugina M, Bonfils P, et al. Evaluation of calcium alginate nasal packing (Algosteril) versus Polyvinyl acetal (Merocel) for nasal packing after inferior turbinate resection. Rhinology. 2006; 44: 58-61.

6. Titiz A, Zeyrek T, Ozcan M, et al. The effects of merocel and glove finger tampon applications on the nasal septum mucosa of rabbits. Rhinology. 2008; 46: 112-115.

7. Stankiewicz JA. Complications of endoscopic sinus surgery and malpractice. In: Stankiewicz JA, ed. Advanced Endoscopic Sinus Surgery. St. Louis: Mosby Year Book, 1995: 151-160.

8. Thaler ER. Postoperative care after endoscopic sinus surgery. Arch Otolaryngol Head Neck Surg. 2002; 128: 1204-1206.

9. Stankiewicz JA. Comments about postoperative care after endoscopic sinus surgery. Arch Otolaryngol Head Neck Surg. 2002; 128 : 1207-1208.

10. Bugten V, Nordgard S, Skogvoll E, et al. Effects of nonabsorbable packing in middle meatus after sinus surgery. Laryngoscope. 2006; 116: 83-86.
11. Gaskins RE. Scarring in endoscopic ethmoidectomy. Am J Rhinol. 1994; 8: 271-274.

12. Weber R, Keerl R, Hochapfel F, et al. Packing in endonasal surgery. Am J Otolaryngol. 2001; 22: 306-320.

13. Weber R, Hochapfel F, Draf W. Packing and stents in endonasal surgery. Rhinology. 2000; 38: 49-62.

14. Fairbanks DNF. Complications of nasal packing. Otolaryngol Head Neck Surg. 1986; 94: 412-415.

15. Weitzel EK, Wormald PJ. A scientific review of middle meatal packing/stents. AJ Rhinol. 2008; 22: 302-307.

16. Baumann A, Caversaccio M. Hemostasis in endoscopic sinus sugery using a specific gelatin-thrombin based agent (FloSeal). Rhinology. 2003; 41: 244-249.

17. Shoman N, Gheriani H, Flamer D, et al. Prospective, double-blind, randomized trial evaulating patient satisfaction, bleeding, and wound healing using biodegradable synthetic polyurethane foam (NasaPore) as a middle meatal spacer in functional endoscopic sinus surgery. J Otolaryngol Head Neck Surg. 2009; 38: 112-118.

18. Chandra RK, Conley DB, and Kern RC. The effect of Floseal on mucosal healing after ESS: A comparison to thrombin-soaked gelatin foam. Am J Rhinol. 17: 51-55, 2003.

19. Chandra RK, Conley DB, Haines GK III, et al. Long-term effects of FloSeal packing after endoscopic sinus surgery. Am J Rhinol. 2005; 19: 240-243

20. Miller RS, Steward DL, Tami TA, et al. The clinical effects of hyaluronic acid ester nasal dressing (Merogel) on intranasal healing after functional endoscopic sinus surgery. Otolaryngol Head Neck. 2003; 128: 862-869.

21. Robinson S, Adams D, Wormald PJ. The effects of nasal packing and prednisolone on mucosal healing and reciliation in a sheep model. Rhinology. 2004; 42: 68-72.

22. Mclntosh D, Cowin A, Adams D, Wormald PJ. The effect of an expandable polyvinyl acetate (Merocel) pack on the healing of the nasal mucosa of sheep. Am J Rhinol. 2005: 19; 577-581.

23. Ruddy J, Brain D, Sudesh RR, Anand $\mathrm{VT}$. A prospective trial of merocel packs. Rhinology. 1991; 29: 281-285.

24. Samad I, Stevens HE, Maloney A. The efficacy of nasal septal surgery. J Otolaryngol. 1992; 21: 88-91.

Amin R. Javer

St Paul's Sinus Centre

1081 Burrard Street

Vancouver

British Columbia

V6Z 1Y6

Canada

Tel: +16048069926

Fax: +16048069690

E-mail: sinussurgeon@drjaver.com 